\title{
Small Bowel: From Sideline to Center Stage in Gastroenterology
}

The small bowel is not considered a central field in gastroenterology. However, celiac disease is the most frequent small intestinal disease in most populations of the world. It is characterized by intolerance to gluten, which causes damage to the resorptive mucosa, ranging from mild enteropathy, with only an increase in intraepithelial lymphocytes and crypt hyperplasia, to severe lesions, characterized by subtotal to total villous atrophy. Although being recognizable macroscopically by the experienced endoscopist, significant villous atrophy requires histological confirmation. However, other causes of villous atrophy, such as giardiasis, autoimmune enteropathy, combined variable immunodeficiency, some drugs, untreated HIV infection, or tropical sprue should be excluded. Notably, endoscopists cannot recognize an early MARSH 1 or MARSH 2 lesion.

Thus, celiac disease of the proximal small bowel, but also inflammatory diseases of the distal small bowel are key to important differential diagnoses of superficially unrelated (extraintestinal) diseases. Small bowel exploration has been facilitated since the early 2000 thanks to the availability of video capsule endoscopy, double-balloon enteroscopy, confocal endomicroscopy and magnetic resonance contrast imaging. Reports about small bowel diseases on many fronts continue to move so fast that a
FALK symposium on this topic seemed mandatory to provide an update and evidence-based authoritative guidelines for an improved clinical practice and translational research. In this issue of Digestive Diseases, a community of internationally renowned experts in gastroenterology, celiac disease and small bowel research have summarized and highlighted advances in the understanding of intestinal immunology, the role of gut microbiota, the pathogenesis and treatment of celiac disease and its extraintestinal manifestations, nonceliac gluten sensitivity and important differential diagnoses.

It is only a short while ago that with the advent of the gluten-free diet (Willem Karel Dicke, Utrecht) in the late 1930s, the duodenal/jejunal biopsies in the 1950s and sensitive and specific serological tests in the 1980s and 1990s, it was realized that most cases of idiopathic steatorrhea were in fact celiac disease in adults and that gut symptoms were not always present in patients who were affected by this disorder. Thus, clinicians are now increasingly recognizing that the majority of patients lack the so-called typical picture of celiac disease, i.e. diarrheal illness with frequent bulky stools, anemia and weight loss. Instead, patients often present with atypical extraintestinal symptoms and coexisting autoimmunity like thyroiditis or type 1 diabetes. 
Celiac disease has also been recognized as a condition that requires (adjunctive) nondietary, i.e. pharmacologi$\mathrm{cal}$, therapy since it is very difficult to maintain a glutenfree diet in daily life because refined food may contain traces of gluten, and many patients do not reach remission even on a perceived strict gluten-free diet.

Refractory celiac disease, especially type 2 , and the advanced stage of intestinal $\mathrm{T}$ cell lymphoma remain rare but difficult to treat and often deadly complications of celiac disease. But progress towards a potentially curative therapy including bone marrow transplantation or biologicals is being made.

Nonceliac gluten sensitivity, a diagnosis made after exclusion of celiac disease or wheat allergy, mainly manifests with worsening of extraintestinal diseases and is being recognized as a separate disease entity apparently due to innate immunity to wheat components.

Next to these topics, the clinical translational knowledge in this issue covers the basic small intestinal immunology, the role of the intestinal microbiome, relevant differential diagnoses, such as food allergies, eosinophilic enteritis, nonceliac gluten sensitivity, FODMAP intolerance, drug-induced inflammation like olmesartan enteropathy, Whipple's disease and motility disorders. We focus in this issue also on symptoms related to celiac disease, the epidemiology not only in the West but also in the African, South American and Asian subcontinents, preventive measures, nutrition and comorbidities of celiac disease such as microscopic colitis, extraintestinal autoimmunity, gluten ataxia and other neurological mani- festations. Finally, we cover new diagnostic tools in serology, genetics and endoscopy, and we cover imaging techniques such as double balloon, capsule endoscopy and MRI endoscopy.

We hope that the proceedings of the FALK symposium of 4-5 September 2014 on Celiac Disease and Other Small Bowel Disorders will help to expand your knowledge and provide you with the best diagnostic and therapeutic tools currently available to manage small bowel disease in general and celiac disease in particular, and will further stimulate your interest in this growing research and clinical field.

This issue is also intended to enhance awareness amongst hepatogastroenterologists of celiac disease and other small intestinal disorders, which are more common than inflammatory bowel diseases and at least as common as Barrett's esophagus. If their experience does not accord with this, they should do biopsy diagnosis and imaging. We feel that a higher awareness and a lower threshold to suspect and clearly diagnose small bowel disease are extremely important to improve diagnostics compared to what we did in the 1990s and 2000s. We hope that this issue of Digestive Diseases will also serve as a handbook for clinicians and researchers dealing with these diseases. The assembly of this issue would not have been possible without the invaluable input of the experts, the unrestrained support of the Falk Foundation and the editing staff of S. Karger AG.

Chris J. Mulder, Amsterdam Detlef Schuppan, Mainz 